This item was submitted to Loughborough's Research Repository by the author.

Items in Figshare are protected by copyright, with all rights reserved, unless otherwise indicated.

\title{
Virtual modelling of a prosthetic foot to improve footwear testing
}

PLEASE CITE THE PUBLISHED VERSION

http://dx.doi.org/10.1243/14644207JMDA82

\section{PUBLISHER}

Sage (@ Institution of Mechanical Engineering)

\section{VERSION}

SMUR (Submitted Manuscript Under Review)

\section{PUBLISHER STATEMENT}

This work is made available according to the conditions of the Creative Commons Attribution-NonCommercialNoDerivatives 4.0 International (CC BY-NC-ND 4.0) licence. Full details of this licence are available at: https://creativecommons.org/licenses/by-nc-nd/4.0/

\section{LICENCE}

CC BY-NC-ND 4.0

\section{REPOSITORY RECORD}

Mara, Gillian E., Andy R. Harland, and Sean R. Mitchell. 2019. "Virtual Modelling of a Prosthetic Foot to Improve Footwear Testing". figshare. https://hdl.handle.net/2134/15717. 
Virtual modelling of a prosthetic foot to improve footwear testing

G E Mara, A R Harland, and S R Mitchell

Mechanical and Manufacturing Engineering, Loughborough University, Loughborough, Leicestershire, UK.

Abstract: The footwear industry is continually producing more technically engineered shoes, therefore, it is necessary to improve existing laboratory footwear tests using simplistic rigid stamps to something more realistic. The aim of this article is to investigate the possibility of reverse engineering a standard commercially available component accurately enough to produce constructive results in a finite-element analysis (FEA). A prosthetic foot was chosen as it is commercially available and is more representative of a real foot. Information on its geometry and material properties were gathered using a non-destructive method. X-ray images and threedimensional laser scanning were used to capture the dimensions of the internal and external geometries, whereas the vickers microhardness test and volume and mass calculations were used along with the Cambridge Engineering Selector software to identify material properties. To validate the finite-element prosthetic foot, a vertical heel compression and a forefoot flexibility laboratory test were conducted and mimicked in an FEA software package. Good and fair agreements were found in the two tests, respectively. It is concluded that a non-destructive approach to reverse engineer a standard component is an effective method of improving the realism of existing footwear tests both in reality and in finite-element situations.

Keywords: finite-element analysis, prosthetic foot, validation, footwear testing

\section{INTRODUCTION}

The footwear industry is continually producing more technically engineered cushioning systems [1] for new footwear designs. To understand how the footwear performs and whether or not the cushioning system achieves the expectations or functions it was designed for, there is a need to test the footwear. Currently, a variety of different controlled laboratory mechanical tests and subject tests, where biomechanical measurements can be a valuable source of information, are used to ascertain how the cushioning systems fully perform. In the laboratory, simple-shaped rigid stamps that are easily manufactured are used to produce a desired change in the shoe in response to an input, which can either be transferred through the stamp or from outside the stamp. For example, rigid heel and forefoot stamps are used to apply a vertical force to the respective areas of the shoe to test the cushioning property. Two upward forces and one downward force are simultaneously used in a three-point bend test arrangement to determine the flexibility property of the shoe in the forefoot area. These three loading conditions are considered to replicate various components of a physical foot strike such as the respective vertical ground reaction 
force component to either the heel or the forefoot [2-4]. The foot strike refers to the stance phase of the gait cycle, i.e. when the foot is in contact with the ground. It is broken down into various stances, i.e. initial landing, midstance, and toe-off [5]. As the complexity of cushioning systems advances, there is a need to increase the realism of the current footwear laboratory tests while maintaining the high repeatability required of a laboratory test. As technology advances, engineering tools that have been used for other applications are being utilized in footwear testing. One such tool is using finite-element analysis (FEA) [6]. This is a computeraided engineering technique that provides a simulated prediction of the mechanical behaviour of an object or structure under loading [7]. In footwear testing, a computer simulation of the shoe could be created and various loading conditions applied; from here on, this will be termed as a virtual test. There are two ways of improving footwear tests. First, existing laboratory-based mechanical tests could be used in conjunction with the development of new loading conditions. The new loading conditions could focus on other components of a foot strike, but still use simple rigid stamps as the effector. Secondly, virtual tests of the existing laboratory mechanical tests could be produced that mimicked the same loading conditions. In reality, the laboratory mechanical tests are limited with what can be achieved mechanically by comparison with the possibilities of virtual testing. However, the challenge remains in ensuring the validity of any virtual test that cannot be replicated in the laboratory. New loading conditions could also be created by modifying the already existing stamps or using more realistic components instead, such as a prosthetic foot. Prosthetics was originally developed for both function and cosmetic appearance and is readily available. Some of the prosthetic feet, available in the market, share mechanical characteristics similar to that of a human foot, such as the solid ankle cushion heel foot created in the 1960s [8]. Other characteristics can include flexion and compression as well as aesthetically pleasing characteristics such as texture and geometry. A prosthetic foot could provide an increased realism and new dimension to footwear tests. The question then arises, is it possible to reverse engineer a standard commercially available component in a non-destructive analysis with sufficient accuracy to allow constructive results to be generated in FEA. This article aims to explain such a possibility and answer this question. In order to successfully virtually represent the prosthetic foot, the internal and external geometries, material properties, and construction must be established. This virtual representation will be known from here on as the virtual model.

\section{GEOMETRY}

\subsection{Internal}

X-ray images of the prosthetic foot were taken. Images were recorded in both the sagittal and transverse planes to identify the shape and dimensions of the internal structure, as shown in Fig. 1. The sagittal plane is the plane that runs from left to right and the transverse plane runs from head to toe. From the images, it was clear that the internal structure was symmetrical in the sagittal plane. In the sagittal plane, 
a reference length was known and this enabled the dimensions of the structure in this plane and the transverse plane to be deduced and scaled to depict a threedimensional drawing. A close inspection of the horizontal plane X-ray image revealed the presence of a pin connecting what will be known from here on as the 'inner bottom plate' and the 'inner top' as shown in Fig. 1. A single three-dimensional CAD model representing the geometry of all constituent components making up the inner was constructed. Its surface was then meshed using a two-dimensional triangular element mesh and its volume meshed using a three dimensional tetrahedral mesh. Owing to the nature of geometry capture used in the formation of the inner model, a number of computationally inefficient tetrahedral elements that were either extremely small or contained very small minimum internal angles were present. The model was, therefore, refined to separate the inner bottom plate and inner top into two discrete components. This process reduced the number of elements used in the inner geometry by 7.7 per cent.

\subsection{External}

The external geometry of the prosthetic foot was captured using a three-dimensional laser scanner. Every fourth three-dimensional point was used to reduce the amount of captured information; this was a compromise between level of detail and points manageable to work with and this process yielded a cloud of 5000 three-dimensional points, as shown in Fig. 2. Although, in general, the external surface of the prosthetic foot is a smooth continuous one replicating a human foot, a cavity exists in the heel region into which a choice of three foam cylinders providing soft, medium, or hard cushioning is inserted, as identified in Fig. 1. To avoid unnecessary complexity, this cavity was not included in the modelled geometry. Splines constructed from the captured surface points were used to create surfaces representing the external geometry of the foot. The default function in the meshing package Altair Hypermesh 6.0 was used to construct the splines. These surfaces were linked together and meshed using a two-dimensional triangular mesh. The one part internal twodimensional triangular mesh of the internal structure was then imported and aligned using the X-ray images to create the interior surface for the external (foam) component. The two two-dimensional mesh surfaces were then adjusted and repaired so that one complete surface was created. It was then possible to create a three-dimensional tetrahedral mesh representing the external component, which could be used in an FEA model. The real prosthetic foot was very detailed in the forefoot region where each toe was individually defined including toenails and, consequently, the external geometry reflected this. This amount of detail requires a number of smaller elements, which are computationally inefficient. The model was therefore refined by smoothing out these features, which allowed larger elements to form the two-dimensional triangular mesh surface. This process reduced the number of elements in the external geometry by 93.9 per cent. A comparative study suggested that the removal of this detail did not significantly affect the mechanical properties of the foot, yet the increase in processing time efficiency was considerable. 
The internal and external geometries in the three-dimensional tetrahedral format were aligned and tied together within the ABAQUS FEA software.

\section{IDENTIFICATION OF MATERIALS}

\subsection{Internal}

An initial survey by sight and touch of the inner material deduced that it was a stiffer material than the outer. A Vickers microhardness test was chosen to gather some of the mechanical characteristic properties of the inner material. The Vickers microhardness test was chosen for a number of reasons. One of the main reasons was the type of indenter: the test uses a diamond indenter in the form of a right pyramid. The pyramid shape allows for greater control of indenter placement into the material, compared with other indenter shapes. The second major reason for the test is the accuracy of the results the test provides. The two diagonals left in the surface of the material from the indenter once it has been removed are carefully measured using a microscope and their average calculated, which achieves accurate results. The microhardness test uses a microscopic scale and, consequently, has higher precision instruments and can operate at loads under $1 \mathrm{kgf}$ [9]. Overall, the Vickers microhardness test was chosen for the type of indenter and accuracy of results. A small sample of the inner material was taken so that the rest of the prosthetic foot could be preserved. The sample was then prepared so that it had a metallographic finish and was enclosed within a resin shell to enable a flat surface, which, in turn, would enable results from the Vickers microhardness test to be accurate. The sample was also investigated under a microscope and longitudinal and transverse fibres were observed, as shown in Fig. 3. These observations were consistent with the traits of a carbon fibre. The results of the Vickers microhardness test applied to the fibres within the material gave an average of $52.3 \mathrm{HV}$ for transverse fibres and 40.75 HV for longitudinal fibres. Using the Cambridge Engineering Selector (CES) software, a specific carbon fibre with a hardness range between 40 and $50 \mathrm{HV}$ was identified. The Young's modulus, the Poisson ratio, and the density of this specific carbon fibre were assigned to the inner material in the model. The material properties that were assigned to the internal material do not account for non-linear behaviour. When the internal material is used for its actual purpose, large deformations of the internal geometry are not observed. Using this assumption, it was assumed that the internal material will stay in the linear phase of the loaddeformation curve. Further justification was shown in the results of the virtual tests, when nominal strain was found to be negligible in the internal geometry.

\subsection{External}

In order to identify and characterize the materials used in the external components, the first parameter that was investigated was density. A range of densities of the external material were derived through simple analyses of the masses and volumes of each of the soft, medium, and hard foam inserts. These densities, together with 
the microscopic analysis of the material structure, as shown in (Fig. 4) and a journal publication [10] written on the foot that provided additional information, allowed the identity of the material to be established as a polyurethane foam. As with the inner, the CES software was used to identify a specific polyurethane foam with a density similar to that found from the inserts. The Poisson ratio and Young's modulus of this foam were then assigned to the material used in the external component of the model. Foams are well known for their highly non-linear behaviour. However, the external material was assigned a linear elastic material model. This was because the deformation imparted on the prosthetic foot throughout testing was small enough to allow the assumption to bemade that the externalmaterial was being strained within its elastic region. Additionally, this kept the modelling to a simplistic and computationally efficient level, allowing much faster analysis run times. If the prosthetic foot was used in mechanical tests other than those stated in this article which involve larger deformations, the material may need to be further developed.

\section{VALIDATION OF THE VIRTUAL MODEL}

The FEA software package used to construct the virtual models was ABAQUS, version 6.5-1. Once the respective material properties were assigned to the appropriate geometries in the ABAQUS workspace, a series of tests were implemented on both the virtual and the real foot to allow validation of the virtual model. Two such tests were a vertical compression test and a forefoot flexibility test.

\subsection{Vertical compression}

A vertical compression with a maximum load of $2000 \mathrm{~N}$ was applied to the heel region of the prosthetic foot, as shown in Fig. 5(a). To ascertain the extent to which the insert affected the deformation, the test was carried out with each of the inserts in place. The load was applied by a vertical compression test machine at a rate of $0.00167 \mathrm{~m} / \mathrm{s}$ and was consistent throughout each compression. For each insert, an average of five compression results was taken. The loading rate has its limitations as it is significantly slower than real world loading scenarios in footwear. For example, in a running heel to toe foot strike, a peak vertical force can occur in the first $0.05 \mathrm{~s}$ [11], with an average peak force of $2000 \mathrm{~N}$, giving an approximate loading rate of 3.6 $\mathrm{m} / \mathrm{s}[12,13]$. The loading rate used for the vertical compression test is not comparative, but the replication of it in the virtual model is important. This test was then replicated on the virtual model of the foot using an ABAQUS standard FEA. Figure 5(b) shows a comparison of the load-deflection curves generated from the real and the virtual compression tests. The deformation at maximum load and the gradient of the curve between 2000 and $1000 \mathrm{~N}$ were used as quantitative measures to ascertain the agreement between the modelled foot and reality. Adjustments were made to the internal and external materials until the virtual performed close to reality. It was found that the virtual model was within 99, 95.77, and 89.5 per cent in agreement with the soft, medium, and firm deformations at maximum load, respectively. The gradient was taken over a period where the soft, medium, and firm 
curves deviate away from each other. The virtual gradient was $308.7 \mathrm{~N} / \mathrm{mm}$ and the soft, medium, and firm gradients were $262.7,284.8$, and $320.5 \mathrm{~N} / \mathrm{mm}$, respectively. Therefore, showing the virtual curve was in close agreement to each of the real curves, as shown in Fig. 5(c).

\subsection{Forefoot flexibility}

The forefoot flexibility test was performed using three cylinders of the same dimensions as used in the laboratory test. Two were positioned $80 \mathrm{~mm}$ apart from each other under the foot and one positioned at the midpoint directly above, this is a similar set-up to that seen in the Runner'sWorld survey [14], as pictured in Fig. 6(a). A displacement of $15 \mathrm{mmwas}$ applied to the forefoot region by a compression test machine. The test was performed five times and an average of the reaction force was recorded. The test was then replicated on the virtual model of the foot using an ABAQUS explicit FEA. Figure 6(b) shows a comparison of the load-deflection curves generated from the real and the virtual flexibility tests. The load at maximum deformation was used as a quantitative measure to ascertain the agreement between the modelled foot and reality. It was found that the virtual model showed an agreement of 186.2 per cent at the maximum deformation point. It is clearly seen from Fig. 6(b) that the virtual curve does not match reality as closely as it did for the vertical compression test. Between 3 and $6 \mathrm{~mm}$, the virtual gradient was $14.5 \mathrm{~N} / \mathrm{mm}$, which almost mirrors the real gradient of $14.1 \mathrm{~N} / \mathrm{mm}$, but the load is underestimated. After $6 \mathrm{~mm}$, the virtual curve shows a larger gradient than the real, 49.8 and 14.1 $\mathrm{N} / \mathrm{mm}$, respectively. The adjusted inner and external material properties were used in the virtual model.

\section{DISCUSSION}

The virtual model of the prosthetic foot was subjected to two virtual tests: the vertical compression test and the flexibility test. Good agreement was shown in the vertical compression test, in which both the maximum deformation point and the gradient were close to the real laboratory test results. Some agreement was also seen in the virtual forefoot flexibility test, in which between specific deformations, similar gradients were found. The agreement does provide some evidence that the geometry and material properties assigned to the internal structure and external component were similar to those of the real prosthetic foot. The difference in agreement between the two virtual tests, particularly the forefoot flexibility test, is now discussed. Identifying possible reasons for the differences could be attributed to one or more of the following three areas. These areas are the geometry, the material properties, and the loading conditions applied of the virtual model. The implications of improvement will be balanced against the time and cost-effectiveness of the virtual model and how sufficient the virtual model needs to be to increase realism and be used as an engineering tool in footwear testing. A closer inspection of the X-ray images shows a pin that joins the two parts of the internal structure together. In the virtual model, the two parts of the internal structure are tied together. This is likely to 
be a major cause of the stiffer curve seen after $6 \mathrm{~mm}$ in the virtual forefoot flexibility test and, consequently, the larger load at the maximum deformation point. To ascertain exactly how the inner bottom plate and inner top were joined would require a destructive analysis of the prosthetic foot, where a nondestructive one was performed. To model the pin as a deformable part rather than rigid body, the inner bottom plate and inner top geometry would have to be refined, as well as the geometry of the pin found. This refinement to the inner structure would need very small elements, where it has already been noted that very small elements are computationally inefficient. Therefore, this method of gaining better agreement may not be time or cost-effective. A second reason for the reduced agreement could be due to the material properties of the prosthetic foot. Further adjustment to the internal and external material properties may improve agreement in the virtual forefoot flexibility test, but this could be a hindrance to the agreement seen in the virtual vertical compression test. The virtual model shows reasonable agreement with the real prosthetic foot, with the evidence shown in the virtual tests. Although improvements could be made to improve agreement, for example, adjusting the geometries would increase the computation time of each of the virtual tests as the total number of elements would increase as well as the number of very small elements. Thus, weighing the time it would take to improve the virtual model against the difference it would make to the two virtual tests would make it neither costeffective nor computationally efficient. A similar reasoning could be made for adjusting the material properties of the internal structure and the external component. Making no further adjustments to the virtual model would mean the virtual model would not be identical to the real prosthetic foot. The implication of this is that the virtual model remains similar, with a reasonable agreement seen between real and virtual. However, the virtual model of the prosthetic foot would be only one small part of a virtual footwear test. The virtual test would also include other parts such as a shoe. Therefore, as the virtual model of the prosthetic foot has generally shown good agreement, it would be more cost efficient to spend the time refining shoe models to ascertain the shoes performance to a similar level of agreement.

\section{CONCLUSIONS}

The reverse engineering process of a prosthetic foot from real to virtual was achieved with results showing suitable agreement. There was strong agreement shown in the virtual compression test and fair agreement in the virtual flexibility test. The virtual model of the prosthetic foot was deemed suitable when weighed against the implications of improving it and the cost-effectiveness it would have when looking at a larger scale of virtual tests that included virtual shoes. As a simple, quick method of improving the realism of existing footwear testing, a prosthetic foot has been demonstrated to be a useful tool for use in real and finite-element situations. 


\section{REFERENCES}

1 Frederick, E. C. The running shoe: dilemmas and dichotomues in design. In The shoe in sport (Eds B. Segesser and W. Pfo“rringer), 1989 (Wolfe Publishing, Ltd, London).

2 Cavanagh, P. R. and Lafortune, M. A. Ground reaction forces in distance running.

J. Biomech., 1980, 13, 397-406.

3 Frederick, E. C., Hagy, J. L., and Mann, R. A. The prediction of vertical impact force during running. J. Biomech., 1981, 14, 498.

4 Frederick, E. C. and Hagy, J. L. Factors affecting peak vertical ground reaction forces. Int. J. Sports Biomech., 1986, 2, 41-49.

5 Adrian, M. J. and Cooper, J.M. Biomechanics of running. In Biomechanics of human movement (Eds M. J. Adrian and J. M. Cooper), 2nd edition, 1995, pp. 295314 (WCB McGraw-Hill).

6 Nishiwaki, T. and Nakabe, N. Numerical design of running shoes based on human response. The engineering of sport 4, (Eds S. Ujihashi and S. J. Hoake), 2002, pp. 393-399 (Blackwell Publishing).

7 Lewis, P. E. and Ward, J. P. The finite element method: principles and applications, 1991 (Addison-Wesley, Wokingham).

8 Ohio Willow Wood. 2005, Available from http://www.owwco.com/aboutus.aspx, accessed 21st October 2005, last updated October 05.

9 England, G. Vickers hardness test, 2005, available from http://www.gordonengland.co.uk/hardness/vickers.htm, accessed 24th October 2005, last updated October 2005.

10 Arbogast, R. and Arbogast, C. R. The carbon copy II - from concept to application. J. Prosthet. Orthot., 1989 1(1), 32-36.

11 Cavanagh, P. R. and Lafortune, M. A. Ground reaction forces in distance running, J. Biomech., 1980, 13, 397-406.

12 Cavanagh, P. R., Andrew, G. C., Kram, R., Rodgers, M. M., Sanderson, D. J., and Hennig, E. M. An approach to biomechanical profiling of elite distance runners. Int. J. Sports Biomech., 1985, 1, 36-62.

13 Freychat, P., Belli, A., Carret, J., and Lacour, J. Relationship between rearfoot and forefoot orientation and ground reaction forces during running. Med. Sci.Sports Exerc., 1996, 28(2), 225-232.

14 Runner's World. Runner's world autumn shoe buyer's guide. Runner's World, September 2005, pp. 52-59. 



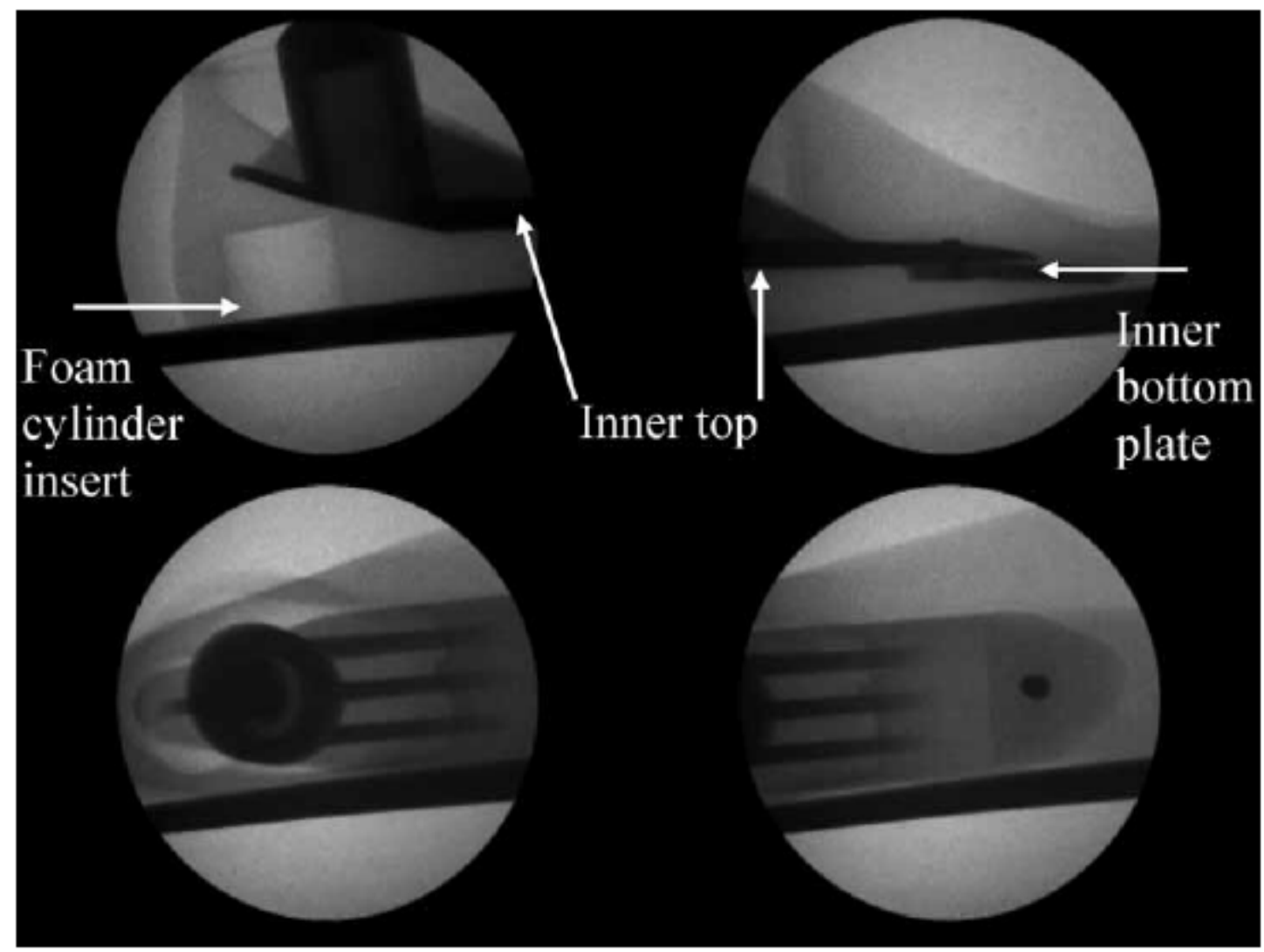

Fig. 1 X-ray images of inner geometry of the prosthetic foot

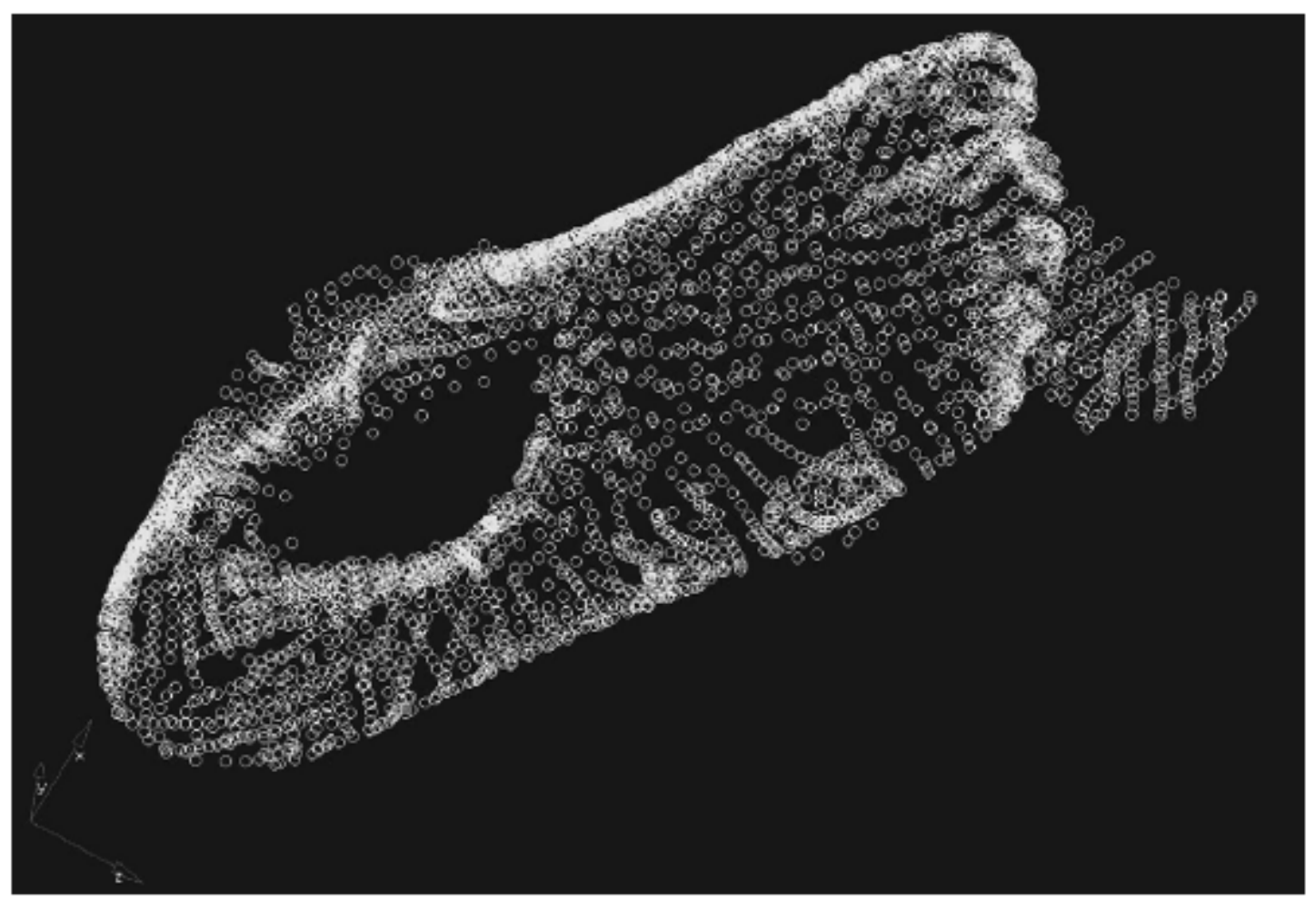

Fig. 2 Three-dimensional laser scan of external geometry of the prosthetic foot 


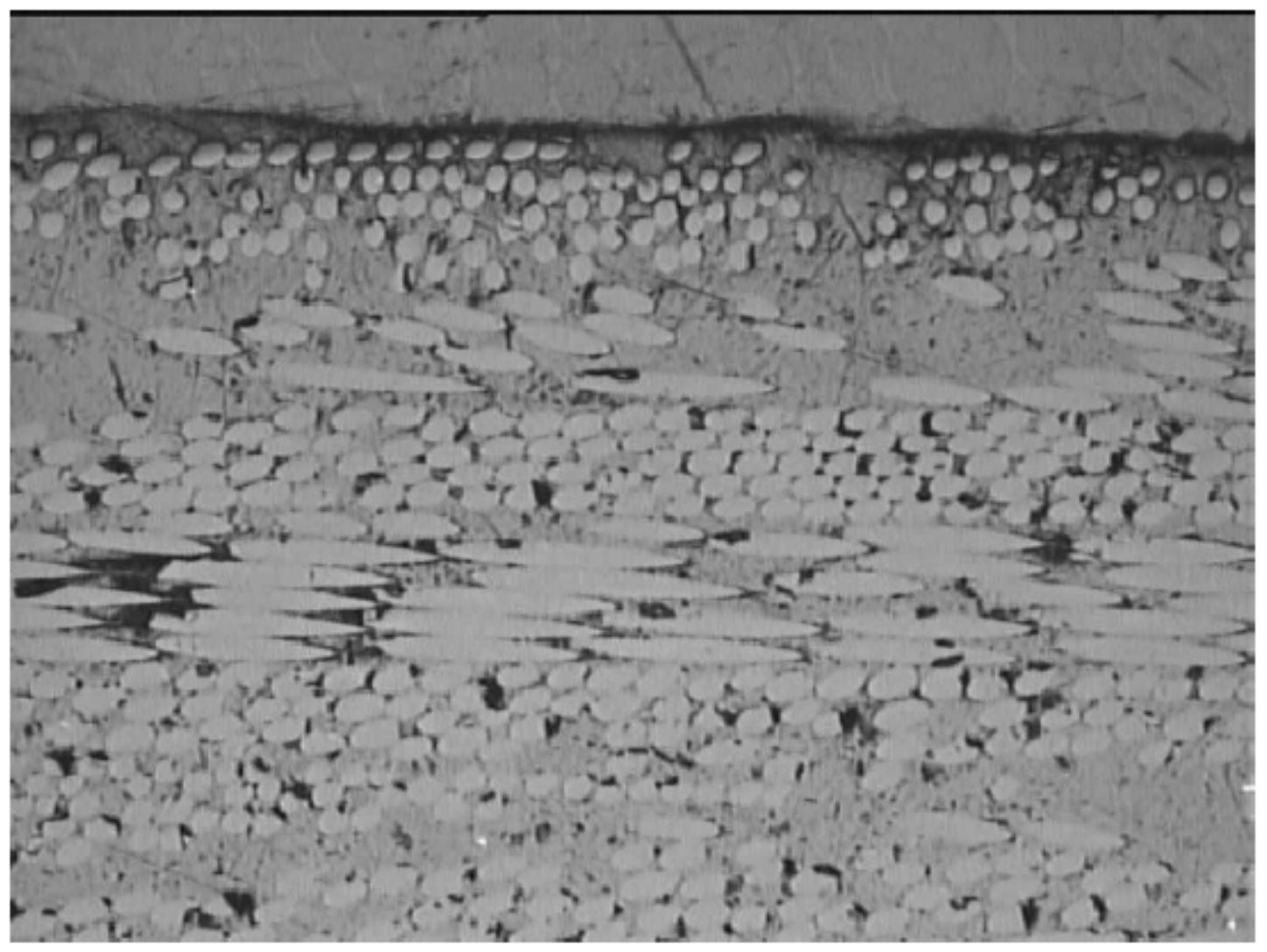

Fig. 3 10x magnification of the internal material

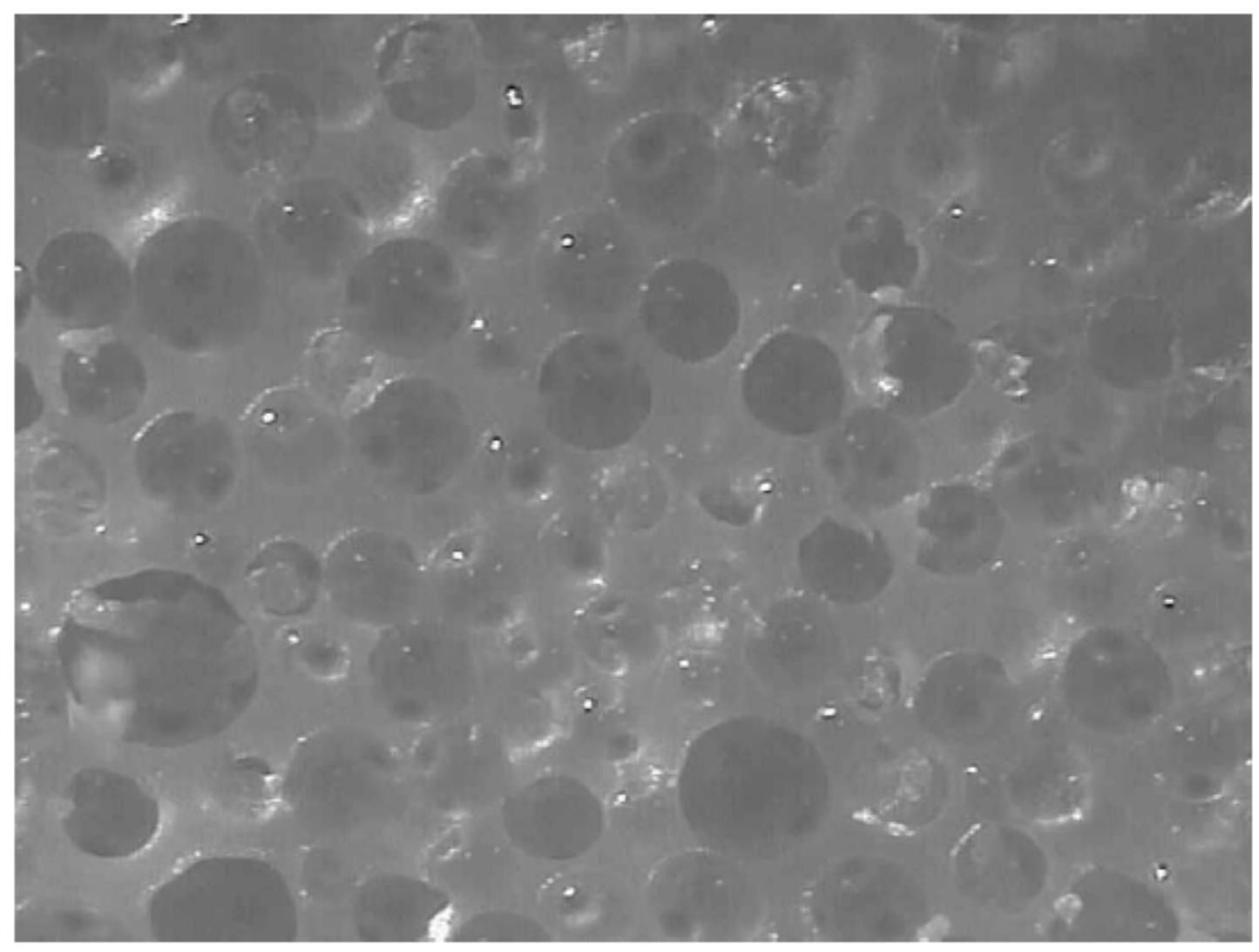

Fig. $45 x$ magnification of the external material 

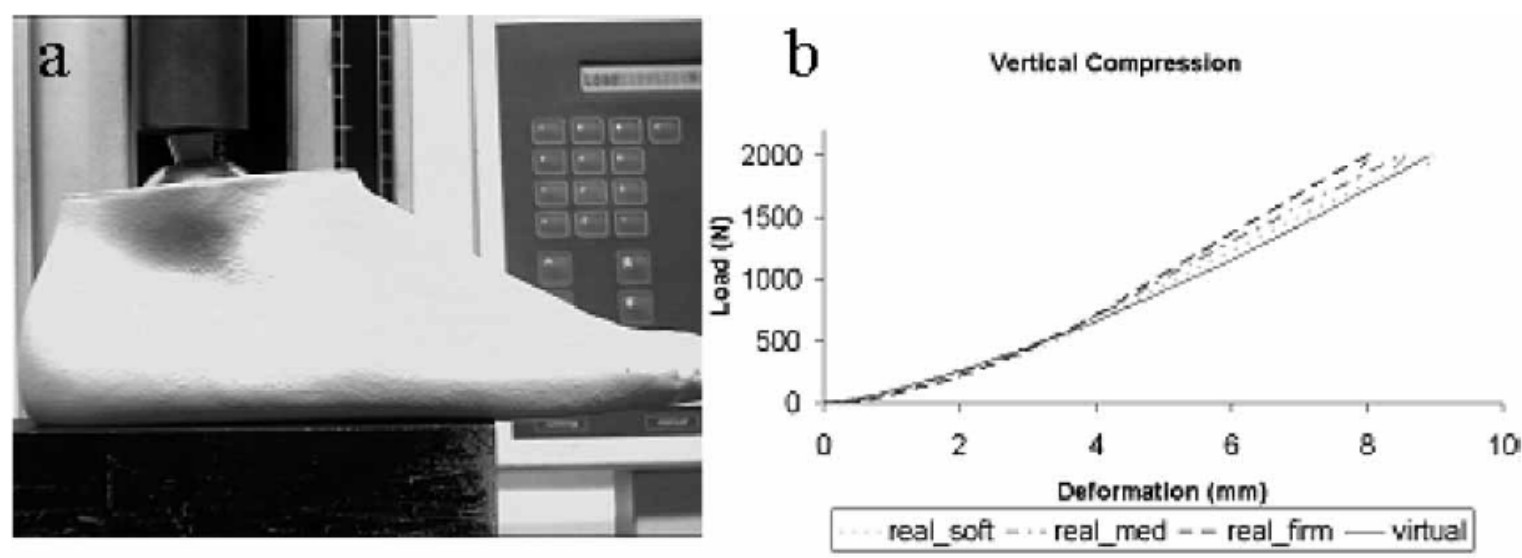

c

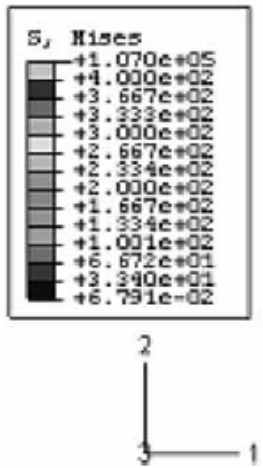

Fig. 5 Comparison of real with virtual vertical compression test
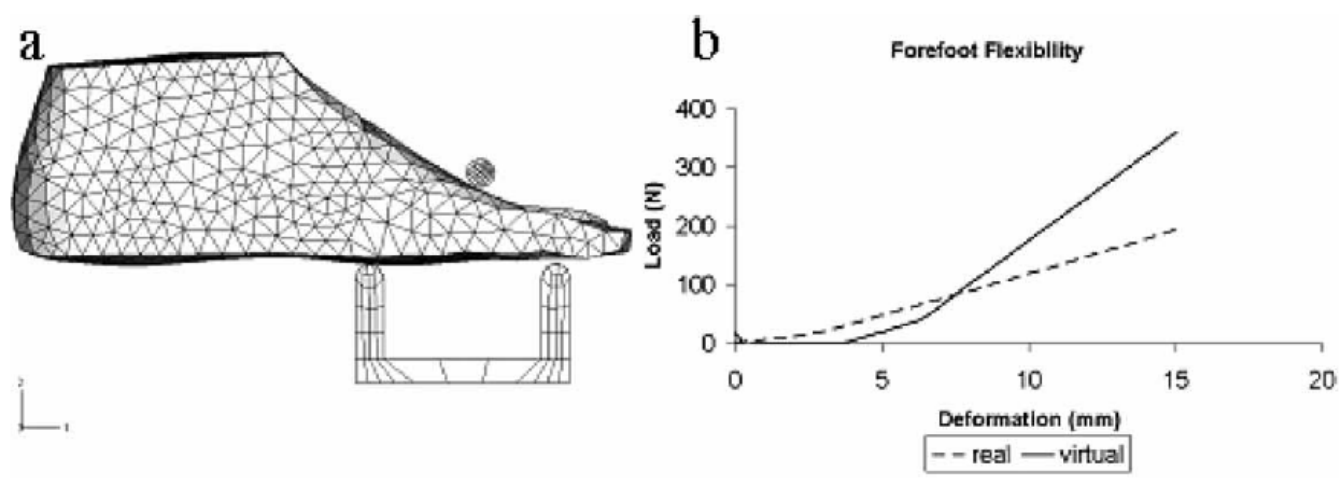

Fig. 6 Forefoot flexibility load-deformation graph 